\title{
Community-Acquired Clostridium difficile Infection: An Emerging Problem
}

\author{
Lenora Lee $\cdot$ Stuart H. Cohen
}

Published online: 5 July 2013

(C) Springer Science+Business Media New York 2013

\begin{abstract}
The incidence of Clostridium difficile infection (CDI) has been increasing in recent years with a trend towards higher hospitalization and mortality rates. Wellknown risk factors such as age and antimicrobial usage have been known to play a role in the development of both hospital-acquired and community-acquired CDI but other potentially novel risk factors such as proton pump inhibitor usage, close contact with a colonized individual and zoonotic or foodborne transmission may contribute to community-acquired cases. There also have been new developments in management of CDI with the use of donor feces for treatment of those with recurrent disease and in the use of probiotics as a strategy for preventing CDI.
\end{abstract}

Keywords Clostridium difficile $\cdot$ Spores - Antimicrobial · Risk factors · Diarrhea

\section{Introduction}

Clostridium difficile is a spore-forming gram-positive anaerobic bacillus that was first detected in 1935 [1••]. Disease occurs through the ingestion of spores, which resist the acidity of the stomach and germinate into the vegetative form in the small intestine $[1 \bullet \cdot$. C. difficile infection (CDI) is caused by two exotoxins, toxin A and B that cause colonic dysfunction and cell death [2]. These two toxins are encoded by their genes, tcdA and tcdB, which are present in the pathogenicity locus (PaLoc) with three regulatory genes. Nontoxigenic strains of $C$. difficile are those that do not

L. Lee $\cdot$ S. H. Cohen $(\varangle)$

Division of Infectious Diseases, UC Davis School of Medicine, 4150 V Street, Suite 500, Sacramento, CA 95817, USA

e-mail: stuart.cohen@ucdmc.ucdavis.edu contain the PaLoc. In recent years, there have been some strains that produce a third toxin, known as a binary toxin that may contribute to disease [1]. CDI ranges in severity from mild diarrhea to fulminant colitis and death. Some patients are colonized with $C$. difficile but do not get the disease. Various factors play a role in development of CDI. First, there must be exposure to the pathogen. Infection is spread via the fecal-oral route with spores able to persist on environmental surfaces for months. Spores of $C$. difficile can be isolated from many sources both inside and outside of the hospital. These spores enable the bacteria to persist in the physical environment for long periods of time, thereby facilitating transmission [3]. After being exposed to the organism, the major risk factor for infection is antimicrobial use due to disruption of the normal gut microbiota, which allows $C$. difficile to grow and produce toxin. Antibacterials disrupt the number and diversity of bacterial populations of the colon that can increase the risk for CDI for 3 months or more [4]. Lastly, host factors such as antibodies to the toxins often determine whether or not colonization is followed by clinical illness and may influence the severity of disease [5].

\section{Epidemiology}

Although primarily thought of as a hospital-acquired infection, CDI in the community is often under-diagnosed. Several studies have described the emergence of community-acquired $C$. difficile infection (CA-CDI). These community-acquired cases are defined as those patients that develop CDI symptoms in the community or $48 \mathrm{~h}$ or less after admission to a health-care facility. These patients must not have been discharged from a health-care facility in the previous 12 weeks [6]. 
During the past decade the epidemiology of CDI has changed significantly. In fact, the incidence, mortality, and medical care costs from CDI have reached historic highs. Between 10 and $25 \%$ of all cases of antibiotic-associated diarrhea in industrialized countries are ascribed to $C$. difficile [7]. From 2000 to 2009, the number of hospitalized patients with CDI as a discharge diagnosis has more than doubled and the number of patients with CDI as the primary diagnosis has more than tripled [4]. In one study by Khanna et al. [8], $50 \%$ of CDI cases were hospitalacquired and $9 \%$ were nursing home-acquired. The increase in the overall number of CDI cases corresponds to the emergence of an epidemic strain known as the NAP1/ $\mathrm{BI} / 027$ strain, which produces higher levels of toxins $\mathrm{A}$ and $\mathrm{B}$ and binary toxin [9].

In addition to hospital-acquired CDI cases, the rate of CA-CDI appears to be increasing. Data from various studies in recent years suggest anywhere from 20 to $45 \%$ of all CDI cases are community associated [1,4]. Given the high rate of antibacterial usage in the community, the high incidence of CDI in outpatients is not unexpected. Interestingly, the CDC also found that a small subset of patients $(5 \%)$ in the outpatient setting have had no previous healthcare exposure [4]. Approximately $22 \%$ of patients have never been exposed to any antimicrobial agents in the 90-day period prior to the diagnosis of CDI [8].

Most patients with CA-CDI present with less severe symptoms than patients with hospital-acquired CDI (HACDI). In a retrospective cohort study by Hirschorn et al. [10], $82 \%$ of the CA-CDI cases were diagnosed and treated exclusively in an ambulatory care setting. Patients with CA-CDI are often younger and more likely to be female compared to patients with HA-CDI [8]. There have been case reports of close contacts to those with $C$. difficile contracting the illness, which may explain this finding [11]. Although CA-CDI is commonly less severe in nature, there have been episodes of severe CDI in populations previously thought to be low-risk. About $10 \%$ of CDI patients diagnosed as outpatients are hospitalized during the course of their illness with low mortality rates in this group of patients $(<3 \%)$ [12]. One other interesting finding noted by Khanna et al. [13] is that the risk of recurrence in patients with CA-CDI and HA-CDI did not differ significantly.

\section{Risk Factors}

Traditionally, the elderly and hospitalized patients who had prior antibacterial therapy were considered to be the most vulnerable to $\mathrm{CDI}$, with this disease regarded as primarily nosocomial for many years [12]. Known risk factors of nosocomial CDI include prior hospitalization, old age
( $>65$ years of age), antimicrobial usage, nasogastric tubes, gastrointestinal surgery, immunosuppression and inflammatory bowel disease [5]. Variability in host factors may also explain the wide spectrum of symptoms and outcome [14•].

There is less information on risk factors for CA-CDI. Similar to HA-CDI cases, known antimicrobial exposure still remains a major risk factor. According to Kuntz et al., the first 30 days after the last antimicrobial exposure are the highest risk for CA-CDI. However the risk continued to remain high until 60 days after exposure and did not return back to baseline until 150 days after antibiotic exposure [15]. The antibiotics most frequently associated with CACDI were fluoroquinolones, cephalosporins and clindamycin. High level resistance to fluoroquinolones is evident in recent isolates of the NAP1/BI/027 strain [16]. This class of antimicrobials is frequently prescribed in the hospitalized setting but may be even more frequently used in the community. Loo et al. [9] postulate that the increased use of newer fluoroquinolones among patients may have promoted the outbreak of a fluoroquinolone-resistant strain in their hospitalized patients. This phenomenon may also be occurring in the outpatient setting, in part accounting for the increased number of CA-CDI cases.

Several case reports have shown that CA-CDI can be acquired in the absence of any known risk factors for this disease and that prior use of antibiotics is not necessary for the development of CDI [5]. Data from various studies shows that this occurs in up to 25-50\% of CA-CDI cases. Thus, other risks for CDI must be implicated in these patients. Studies have shown that outpatient visits are strongly associated with CA-CDI. In addition, contact with those that may be colonized can also be considered a risk factor. Colonization of healthy nonhospitalized patients is uncommon $(<5 \%)$ but colonization among hospitalized patients and especially long-term health-care facility residents can range from 25 to $55 \%$ [1]. Increased $C$. difficile carriage in outpatients could contribute to the risk of CACDI in community dwellers without antimicrobial exposure [13].

Increased age has been associated with a significantly higher risk of CDI after antibiotic exposure, whether in the community or in the hospital. The typical age of community-acquired cases is lower compared to hospital-acquired cases [17]. According to Khanna et al. [8], females were also more likely than men to have CA-CDI. One hypothesis for this is that females are more likely to seek medical attention and thus be exposed to antibiotics in the outpatient setting than their male counterparts.

Some studies have also investigated the risk of proton pump inhibitors (PPIs) with CA-CDI. Hypotheses behind this association are that the ability of spores to reach the small intestine may be facilitated by elevated gastric $\mathrm{pH}$ 
levels. It is also possible that they alter the intestinal microbiota [18]. Inconsistent results between studies has led to a lack of consensus on whether PPIs increase the risk of CDI [19]. In one study by Naggie et al., $15 \%$ of patients met the criteria for CA-CDI. Over one-third of all enrolled patients reported taking a PPI or an $\mathrm{H} 2$ antagonist but this exposure was not associated with CDI [20]. In another study by Lowe et al. [19], elderly outpatients hospitalized for CA-CDI after antibiotic use were no more likely than control subjects to have been treated with PPIs. Although the data linking PPI and $\mathrm{H} 2$ blockers with CDI is not definitive, Loo et al. [14] did find that PPI use was associated with health care-associated colonization and infection. One can theorize that increasing the risk of colonization can lead to an increased risk of CDI cases in the community just as in the hospital.

Another hypothesis for acquisition of $C$. difficile in the community is via zoonotic or foodborne spread. CDI in animals was first described in 1968 when a case of fatal enteritis was described in hamsters [12]. $C$. difficile has been isolated from almost all mammals including cows, horses, pigs, elephants, bears and nonhuman primates in addition to poultry. Studies have shown $C$. difficile to be a pathogen or colonizer of calves, pigs and humans [3]. Pet dogs and cats can be colonized with $C$. difficile. Interestingly, all toxigenic strains from pets have been implicated in CDI [12]. Regarding the controversy of CDI as a zoonotic disease, Hesngens et al. questions whether zoonotic transmission occurs. Studies have not been definitive and there has not been solid evidence to link human and animal exposure as an important reservoir for strains that cause human disease [12].

Foodborne transmission via meat has also been considered as a potential source of CDI with up to $42 \%$ of meat samples being contaminated with $C$. difficile [12]. There has been recent detection of the NAP1/BI/027 strain of $C$. difficile in retail ground beef. Only low levels of contamination were found but the spores' ability to withstand heat may increase the chance of infection. $C$. difficile has also been found in seafood and fish as well as vegetables. It has not been isolated from milk and milk products [12]. Despite the possibility of human consumption via food, foodborne infection has never been confirmed, but this area deserves further study.

\section{Clinical Manifestations}

Community-acquired $C$. difficile infection cases present no differently from hospital-acquired cases in regards to symptoms. The incubation period for CDI is a median of 2-3 days. Those patients who become infected with C. difficile can have colitis without pseudomembrane formation. Patients that fall into the category of mild CDI rarely have systemic symptoms as the toxins do not penetrate the colonic mucosa and therefore cannot enter the bloodstream and cause sepsis [21]. Prominent clinical symptoms include mild to moderate diarrhea, fever, vomiting, abdominal pain and leukocytosis. Fulminant colitis is uncommon in those with CA-CDI. In a VA study performed in North Carolina, the proportion of patients with fever, leukocytosis and renal insufficiency in the case of patients with CA-CDI were lower than the controls with HA-CDI. No case patients were admitted to the ICU for CDI and no one underwent colectomy [17]. In this study, $49 \%$ of the patients were not exposed to antimicrobial drugs.

\section{Diagnosis}

Clinicians should consider the diagnosis of CA-CDI in those outpatients with severe diarrhea even if they lack the traditional risk factors associated with CDI [3]. In elderly patients, given their higher risk of mortality, those with signs and symptoms of severe infection should be monitored closely and managed more aggressively in the community to prevent poor outcomes [13]. To diagnose C. difficile in the community, both CPR assays and the enzyme immunoassay (EIA) for detection of toxins A and $\mathrm{B}$ are used. The EIA test is still the most widely used test because of its rapid turnaround, low cost and simplicity. But PCR assays with higher sensitivities than EIA and are increasingly being adopted. However the disadvantage of using PCR testing is that it may pick up cases of those that are colonized with $C$. difficile but have diarrhea of an alternative etiology. Test of cure is discouraged because the organism and toxins can persist long after symptoms have resolved. As many as $40 \%$ of patients will continue to show positive results in toxin assays up to 6 weeks after resolution of their diarrhea [8].

\section{Treatment}

The mean duration of diarrhea in those patients with CDI is 3-5 days with most cases resolving by day 7 [8]. Given that most cases of CA-CDI are mild in severity, most patients can be treated with metronidazole as below, as indicated by the IDSA Practice Guidelines [22•]:

\begin{tabular}{lll}
\hline & Treatment & Duration \\
\hline $\begin{array}{l}\text { Mild to moderate } \\
\text { CDI }\end{array}$ & $\begin{array}{l}\text { Metronidazole 500 mg PO three } \\
\text { times/day }\end{array}$ & $10-14$ days \\
\hline
\end{tabular}




\begin{tabular}{lll}
\hline & Treatment & Duration \\
\hline Severe CDI & $\begin{array}{c}\text { Vancomycin 125 mg PO four } \\
\text { times/day }\end{array}$ & 10-14 days \\
Severe, & $\begin{array}{l}\text { Vancomycin 500 mg PO four } \\
\text { times/day + vancomycin }\end{array}$ \\
complicated & enemas PR 500 mg every & \\
CDI & $6 \mathrm{~h}+$ metronidazole 500 mg & \\
& IV q8h & Immediate \\
Signs of toxic & Subtotal colectomy sparing & surgical \\
megacolon, & rectum & intervention \\
septic shock & & \\
\hline
\end{tabular}

Treatment of the first recurrence of CDI is usually with the same regimen as for the initial episode but metronidazole is not recommended beyond the first recurrence of CDI [22•]. One of the newer agents being used in recent years for treatment of CDI is fidaxomicin. Fidaxomicin is an antibiotic that is more active in vitro than vancomycin against isolates of $C$. difficile, including NAP1/BI/027 strains. It has minimal systemic absorption but high fecal concentrations and does not disrupt the fecal microbiota as much as metronidazole or vancomycin. In a recent randomized control trial by Louie et al., fidaxomicin $(200 \mathrm{mg}$ twice daily for 10 days) was compared to vancomycin (125 mg four times daily for 10 days). Results showed a $45 \%$ relative reduction in recurrences and an improved rate of global cure with fidaxomicin compared to vancomycin [23•]. In another randomized control study looking at primarily first recurrences of CDI, treatment of an initial recurrence with fidaxomicin was associated with fewer second recurrences than treatment with vancomycin within 28 days of completion of therapy [24]. Overall, there appears to be a more sustained resolution of disease achieved with fidaxomicin; however its use may be limited given its high financial cost.

Discussion of management of patients with multiple recurrences is beyond the scope of this manuscript. But interest has been increasing for the use of fecal microbiota transplantation based in part on a recent study done by van Nood et al. They used oral vancomycin with bowel lavage, followed by the infusion of donor feces via a nasoduodenal tube and showed an overall cure rate of $94 \%$ compared to the $23-31 \%$ cure rate noted in the control groups (oral vancomycin alone or oral vancomycin plus bowel lavage). In addition, there were few adverse events and the infusion of donor feces. Moreover the restored fecal microbiota was that of their donors [25•]. This study was done in a population that was relatively healthy and in those without severe CDI. Thus, it may be a potential treatment option for those outpatients with CA-CDI that have had multiple recurrences of CDI.

\section{Prevention}

Prevention of disease, especially in the outpatient setting can be done with judicious use of antimicrobial prescriptions. About $50 \%$ of all antibiotics given are not needed according to the Centers for Disease Control [4]. Discontinuation of all antimicrobials will potentially allow the normal bowel microflora to restore itself [16]. Recently the idea of using probiotics to help restore or maintain normal bowel flora has been a topic of discussion. Probiotics are microorganisms believed to counteract disturbances in the intestinal flora created by antibiotics, thereby reducing the risk for colonization by $C$. difficile [26]. Lactobacillus species and Saccharomyces boulardii have shown efficacy in reducing the incidence of simple antibiotic-associated diarrhea [16]. A recent meta-analysis by Johnston et al. evaluated 20 randomized trials testing the effect of probiotics in patients receiving antibiotics that showed a statistically significant risk reduction in the incidence of CDI. Side effects reported included abdominal cramping, fever, soft stools and flatulence and none of the trials reported a serious adverse event [26]. They concluded that there now is some evidence supporting the protective effect of probiotics in preventing CDI. However, there are significant limitations of this meta-analysis. The methodology of many of the studies was inconsistent. Moreover 2 or 3 studies were largely responsible for the statistical significance. Additionally several different microorganisms were used as probiotics and the analysis suggested that they all were interchangeable.

\section{Summary}

The incidence of CDIs has been increasing in recent years with the number of community-acquired cases becoming more prevalent and should be considered in patients presenting with a diarrheal illness. This may be in part due to an increased number of prescribed antimicrobials in the outpatient setting, the use of $\mathrm{PPI} / \mathrm{H} 2$ blockers leading to increased colonization, and foodborne or zoonotic transmission. However there are patients with no known risk factors that develop CDI in the community. Cases of CACDI are often less severe in nature and treatment is the same as cases of HA-CDI. New and innovative approaches to these patients include the use of fecal transplantation, a potential consideration for those with recurrent community-acquired cases and the use of probiotics to restore or maintain the normal intestinal microbiota.

Disclosure L/Lee: none; S. Cohen: consultant for Optimer. This article does not contain any studies with human or animal subjects performed by any of the authors. 


\section{References}

Papers of Importance, published recently, have been highlighted as:

- Of importance

•- Of major importance

1. •- Lessa FC, Gould CV, McDonald LC. Current status of Clostridium difficile infection epidemiology. Clin Infect Dis. 2012;55(Suppl 2):S65-70. This article discusses the changes in the epidemiology of CDI in recent years, noting an increased incidence and severity of CDI cases. They bring to light the fact that CDI is being discovered in low-risk populations and that $C$. difficile has the potential to become more virulent in the future due to its highly fluid genome.

2. Centers for Disease Control and Prevention (CDC). Severe Clostridium difficile-associated disease in populations previously at low risk-four states, 2005. MMWR Morb Mortal Wkly Rep. 2005;54(47):1201-5.

3. Centers for Disease Control and Prevention (CDC). Surveillance for community-associated Clostridium difficile-Connecticut, 2006. MMWR Morb Mortal Wkly Rep. 2008;57(13):340-3.

4. Centers for Disease Control and Prevention (CDC). Vital signs: preventing Clostridium difficile infections. MMWR Morb Mortal Wkly Rep. 2012;61(9):157-62.

5. Bauer MP, et al. Community-onset Clostridium difficile-associated diarrhoea not associated with antibiotic usage-two case reports with review of the changing epidemiology of Clostridium difficile-associated diarrhoea. Neth J Med. 2008;66(5):207-11.

6. McDonald LC, et al. Recommendations for surveillance of Clostridium difficile-associated disease. Infect Control Hosp Epidemiol. 2007;28(2):140-5.

7. Beaugerie L, et al. Antibiotic-associated diarrhoea and Clostridium difficile in the community. Aliment Pharmacol Ther. 2003;17(7):905-12.

8. Khanna S, et al. The epidemiology of community-acquired Clostridium difficile infection: a population-based study. Am J Gastroenterol. 2012;107(1):89-95.

9. Loo VG, et al. A predominantly clonal multi-institutional outbreak of Clostridium difficile-associated diarrhea with high morbidity and mortality. N Engl J Med. 2005;353(23):2442-9.

10. Hirschhorn LR, et al. Epidemiology of community-acquired Clostridium difficile-associated diarrhea. J Infect Dis. 1994; 169(1):127-33.

11. Wilcox $\mathrm{MH}$, et al. A case-control study of community-associated Clostridium difficile infection. $\mathrm{J}$ Antimicrob Chemother. 2008;62(2):388-96.

12. Hensgens MP, et al. Clostridium difficile infection in the community: a zoonotic disease? Clin Microbiol Infect. 2012;18(7): $635-45$.

13. Khanna $\mathrm{S}$, et al. Outcomes in community-acquired Clostridium difficile infection. Aliment Pharmacol Ther. 2012;35(5):613-8.

14. - Loo VG, et al. Host and pathogen factors for Clostridium difficile infection and colonization. N Engl J Med. 2011;365(18): 1693-703. This study analyzes various host and pathogen variables contributing to health care-associated $C$. difficile infection and colonization. They determined that age, antimicrobial usage and PPIs were significantly associated with health care-associated infection, with these patients being more likely to be infected with the NAP1 strain than those that were colonized with $C$. difficile.

15. Kandel C, Moayedi Y, Bunce PE. A woman with communityacquired Clostridium difficile infection. CMAJ. 2012;184(12): 1383-5.

16. Kelly CP, LaMont JT. Clostridium difficile-more difficult than ever. N Engl J Med. 2008;359(18):1932-40.

17. Kutty PK, et al. Risk factors for and estimated incidence of community-associated Clostridium difficile infection, North Carolina, USA. Emerg Infect Dis. 2010;16(2):197-204.

18. Dial S, et al. Use of gastric acid-suppressive agents and the risk of community-acquired Clostridium difficile-associated disease. JAMA. 2005;294(23):2989-95.

19. Lowe DO, et al. Proton pump inhibitors and hospitalization for Clostridium difficile-associated disease: a population-based study. Clin Infect Dis. 2006;43(10):1272-6.

20. Naggie S, et al. A case-control study of community-associated Clostridium difficile infection: no role for proton pump inhibitors. Am J Med. 2011;124(3):276 e1-7.

21. Bartlett JG. New drugs for Clostridium difficile infection. Clin Infect Dis. 2006;43(4):428-31.

22. - Cohen SH, et al. Clinical practice guidelines for Clostridium difficile infection in adults: 2010 update by the society for healthcare epidemiology of America (SHEA) and the infectious diseases society of America (IDSA). Infect Control Hosp Epidemiol. 2010;31(5):431-55. These guidelines, published by the SHEA and IDSA, are the most current guidelines regarding epidemiology, diagnosis, and treatment of $C$. difficile infections. The guidelines also discuss prevention in regards to infection control.

23. • Louie TJ, et al. Fidaxomicin versus vancomycin for Clostridium difficile infection. N Engl J Med. 2011;364(5):422-31. This paper discusses the use of a newer agent, fidaxomicin, in treatment of $C$. difficile infections. This study compared oral fidaxomicin to vancomycin and looked at the rates of clinical cure and recurrence of infection. Results found that fidaxomicin was noninferior to vancomycin in terms of clinical cure but there was a significant decrease in recurrence rate in the fidaxomicin group compared to the vancomycin group.

24. Cornely OA, et al. Treatment of first recurrence of Clostridium difficile infection: fidaxomicin versus vancomycin. Clin Infect Dis. 2012;55(Suppl 2):S154-61.

25. - van Nood E, et al. Duodenal infusion of donor feces for recurrent Clostridium difficile. N Engl J Med. 2013;368(5): 407-15. This recently published paper discusses one of the intriguing topics regarding $C$. difficile treatment. This study showed that infusion of donor feces proved to be more effective in curing those with recurrent CDI when compared to standard treatment with vancomycin alone and vancomycin with bowel lavage. Although unconventional, fecal transplant will likely be an important and effective treatment modality in upcoming years.

26. Johnston BC, et al. Probiotics for the prevention of Clostridium difficile-associated diarrhea: a systematic review and meta-analysis. Ann Intern Med. 2012;157(12):878-88. 